\title{
SUPT6H is a differentially expressed gene in brain metastatic human breast cancer.
}

Shahan Mamoor, $\mathrm{MS}^{1}$

${ }^{1}$ shahanmamoor@gmail.com

East Islip, NY USA

Metastasis to the brain is a clinical problem in patients with breast cancer ${ }^{1-3}$. We mined published microarray data ${ }^{4,5}$ to compare primary and metastatic tumor transcriptomes for the discovery of genes associated with brain metastasis in humans with metastatic breast cancer. We found that the suppressor of Ty 6 homolog, encoded by SUPT6H, was among the genes whose expression was most different in the brain metastases of patients with metastatic breast cancer as compared to primary tumors of the breast. SUPT6H mRNA was present at increased quantities in brain metastatic tissues as compared to primary tumors of the breast. Importantly, expression of SUPT6H in primary tumors was significantly correlated with patient distant metastasis-free survival. Modulation of SUPT6H expression may be relevant to the biology by which tumor cells metastasize from the breast to the brain in humans with metastatic breast cancer.

Keywords: breast cancer, metastasis, brain metastases, central nervous system metastases, the suppressor of Ty 6 homolog, SUPT6H, systems biology of breast cancer, targeted therapeutics in breast cancer. 
One report described a $34 \%$ incidence of central nervous system metastases in patients

\section{Methods}

We used datasets GSE10893 ${ }^{4}$ and GSE52604 ${ }^{5}$ for this global differential gene expression analysis of brain metastatic breast cancer in conjunction with GEO2R. GSE10893 was generated using Agilent-011521 Human 1A Microarray G4110A technology with $n=11$ primary breast tumors and $n=3$ brain metastases from patients with breast cancer; analysis was performed using platform GPL885. GSE52604 was generated using Agilent-014850 Whole Human Genome Microarray $4 \times 44 \mathrm{~K}$ G4112F with $n=10$ normal breast tissues and $n=35$ brain metastases from patients with breast cancer; analysis was performed using platform GPL6480. The Benjamini and Hochberg method of $p$-value adjustment was used for ranking of differential expression but raw $p$-values were used to assess statistical significance of global differential expression. Log-transformation of data was auto-detected, and the NCBI generated category of platform annotation was used. A statistical test was performed to evaluate whether SUPT6H gene expression was significantly different between primary tumors of the breast and brain metastases in humans with breast cancer using an unpaired two-tailed t-test with Welch's correction; PRISM 9.0 was utilized for statistical analyses. For Kaplan-Meier survival analysis, we used the Kaplan-Meier plotter online tool ${ }^{7}$ for correlation of SUPT6H mRNA expression levels with distant metastasis-free survival in $n=1803$ breast cancer patients.

\section{$\underline{\text { Results }}$}

We performed global comparative transcriptome analysis of metastatic tumor tissues of patients with metastatic breast cancer using published microarray data ${ }^{4,5}$ to describe the transcriptional landscape of brain metastasis in human breast cancer in an unbiased fashion and for the discovery of novel therapeutic targets.

\section{SUPT6H is differentially expressed in the brain metastases of patients with brain metastatic breast cancer.}

Through blind, systems-level analysis of published microarray $\operatorname{data}^{4}$, we identified the 
suppressor of Ty 6 homolog, encoded by SUPT6H, as a differentially expressed gene in the breast metastatic tissues of humans with breast cancer (Table 1). When sorting each of the genes expressed in brain metastases based on significance of difference as compared to primary tumors of the breast in patients with breast cancer, SUPT6H ranked 162 out of 17418 total transcripts (Chart 1), equating to $99.1 \%$ differential expression. Differential expression of SUPT6H in the brain metastases of patients with metastatic breast cancer was statistically significant (Chart 1; $p=4.06 \mathrm{E}-04)$.

Differential gene expression analyses that utilize primary and metastatic tumor tissues to discover genes associated with metastasis can be challenging due to the relatively heterogenous cellular composition and constitution of the tumor as compared to normal, untransformed (benign) tissues. Thus, to attempt to validate differential transcriptome-wide differential expression of SUPT6H in brain metastases in human metastatic breast cancer, we queried a second microarray dataset ${ }^{5}$, here comparing normal breast tissues rather than primary tumors of the breast to brain metastases. Again, we identified SUPT6H as a differentially expressed gene in the brain metastatic tissues of patients with breast cancer (Chart 2). When sorting each of the genes expressed in brain metastases based on significance of difference as compared to normal breast tissues, SUPT6H ranked 13203 out of 41093 total transcripts (Chart 2), equating to 67.9\% differential expression. Differential expression of SUPT6H in the brain metastases of patients with metastatic breast cancer was statistically significant (Chart $2 ; p=1.66 \mathrm{E}-02$ ). Thus, differential expression of SUPT6H, transcriptome-wide, in the brain metastases of women with metastatic breast cancer was conserved across two independent microarray datasets, both when compared to benign and transformed breast tissues.

\section{SUPT6H is expressed at higher levels in the brain metastases of patients with metastatic} breast cancer.

We obtained exact mRNA expression levels for SUPT6H, in primary tumors of the breast and in brain metastasis of patients with brain metastatic breast cancer to determine direction and statistical significance of change in SUPT6H expression in brain metastatic tissues. SUPT6H was expressed at higher levels in the brain metastases of patients with breast cancer as compared to primary tumors of the breast, and this difference was statistically significant (Figure 1; $p=0.000417)$.

\section{SUPT6H expression is significantly correlated with survival outcomes in human breast} cancer.

We performed Kaplan-Meier survival analysis ${ }^{8}$ in $n=1803$ breast cancer patients in total, to evaluate whether SUPT6H tumor expression was correlated with survival outcomes in breast cancer. We observed a statistically significant correlation between primary tumor expression of SUPT6H and distant metastasis-free survival (DMFS) in patients with breast cancer (Figure 2). Patients whose primary tumors expressed low levels of SUPT6H possessed median DMFS of 118.51 months, while patients whose tumors expressed high levels of SUPT6H possessed median DMFS of 84 months. This difference in DMFS based on SUPT6H tumor expression in patients with breast cancer was statistically significant (Figure 2, Chart 3; logrank $p$-value: 0.03; hazard ratio: $1.24(1.02-1.5))$. 
Thus, by mining published microarray data ${ }^{4,5}$ in an unbiased and systematic fashion, we identified the suppressor of Ty 6 homolog, encoded by SUPT6H, as among the genes whose expression was most different, transcriptome-wide, in the brain metastases of patients with breast cancer, both when compared to primary tumors of the breast and to normal breast tissues; we observed significantly increased expression of SUPT6H in brain metastases as compared to primary tumors of the breast. Further, we found a significant correlation between SUPT6H expression and patient survival outcomes, as distant metastasis-free survival was significantly greater in patients whose primary tumors expressed lower levels of SUPT6H as compared to patients whose primary tumors expressed higher levels of SUPT6H.

\section{Discussion}

We provided evidence here that the suppressor of Ty 6 homolog, encoded by SUPT6H, is among the genes whose expression is most different in the brain metastases of patients with brain metastatic breast cancer, that SUPT6H mRNA is present at significantly increased quantities in brain metastatic tissues as compared to primary tumors of the breast, and that primary tumor SUPT6H expression is significantly correlated with patient survival outcomes in human breast cancer. Evaluation of the effects of genetic depletion of SUPT6H in mouse models of metastatic breast cancer on metastasis to the central nervous system is merited. Modulation of SUPT6H expression may be relevant to the processes by which breast cancer cells exit the breast, enter the vasculature and/or lymphatics, reside in the lymph nodes, evade immune clearance, breach the blood-brain barrier and colonize the brain. 


\section{References}

1. Lin, N.U., Amiri-Kordestani, L., Palmieri, D., Liewehr, D.J. and Steeg, P.S., 2013. CNS metastases in breast cancer: old challenge, new frontiers.

2. Bendell, J.C., Domchek, S.M., Burstein, H.J., Harris, L., Younger, J., Kuter, I., Bunnell, C., Rue, M., Gelman, R. and Winer, E., 2003. Central nervous system metastases in women who receive trastuzumab-based therapy for metastatic breast carcinoma. Cancer, 97(12), pp.2972-2977.

3. Tsukada, Y., Fouad, A., Pickren, J.W. and Lane, W.W., 1983. Central nervous system metastasis from breast carcinoma autopsy study. Cancer, 52(12), pp.2349-2354.

4. Weigman, V.J., Chao, H.H., Shabalin, A.A., He, X., Parker, J.S., Nordgard, S.H., Grushko, T., Huo, D., Nwachukwu, C., Nobel, A. and Kristensen, V.N., 2012. Basal-like Breast cancer DNA copy number losses identify genes involved in genomic instability, response to therapy, and patient survival. Breast cancer research and treatment, 133(3), pp.865-880.

5. Salhia, B., Kiefer, J., Ross, J.T., Metapally, R., Martinez, R.A., Johnson, K.N., DiPerna, D.M., Paquette, K.M., Jung, S., Nasser, S. and Wallstrom, G., 2014. Integrated genomic and epigenomic analysis of breast cancer brain metastasis. Plos one, 9(1), p.e85448.

6. Awada, A., Colomer, R., Inoue, K., Bondarenko, I., Badwe, R.A., Demetriou, G., Lee, S.C., Mehta, A.O., Kim, S.B., Bachelot, T. and Goswami, C., 2016. Neratinib plus paclitaxel vs trastuzumab plus paclitaxel in previously untreated metastatic ERBB2-positive breast cancer: the NEfERT-T randomized clinical trial. JAMA oncology, 2(12), pp.1557-1564.

7. Györffy, B., Lanczky, A., Eklund, A.C., Denkert, C., Budczies, J., Li, Q. and Szallasi, Z., 2010. An online survival analysis tool to rapidly assess the effect of 22,277 genes on breast cancer prognosis using microarray data of 1,809 patients. Breast cancer research and treatment, 123(3), pp.725-731. 


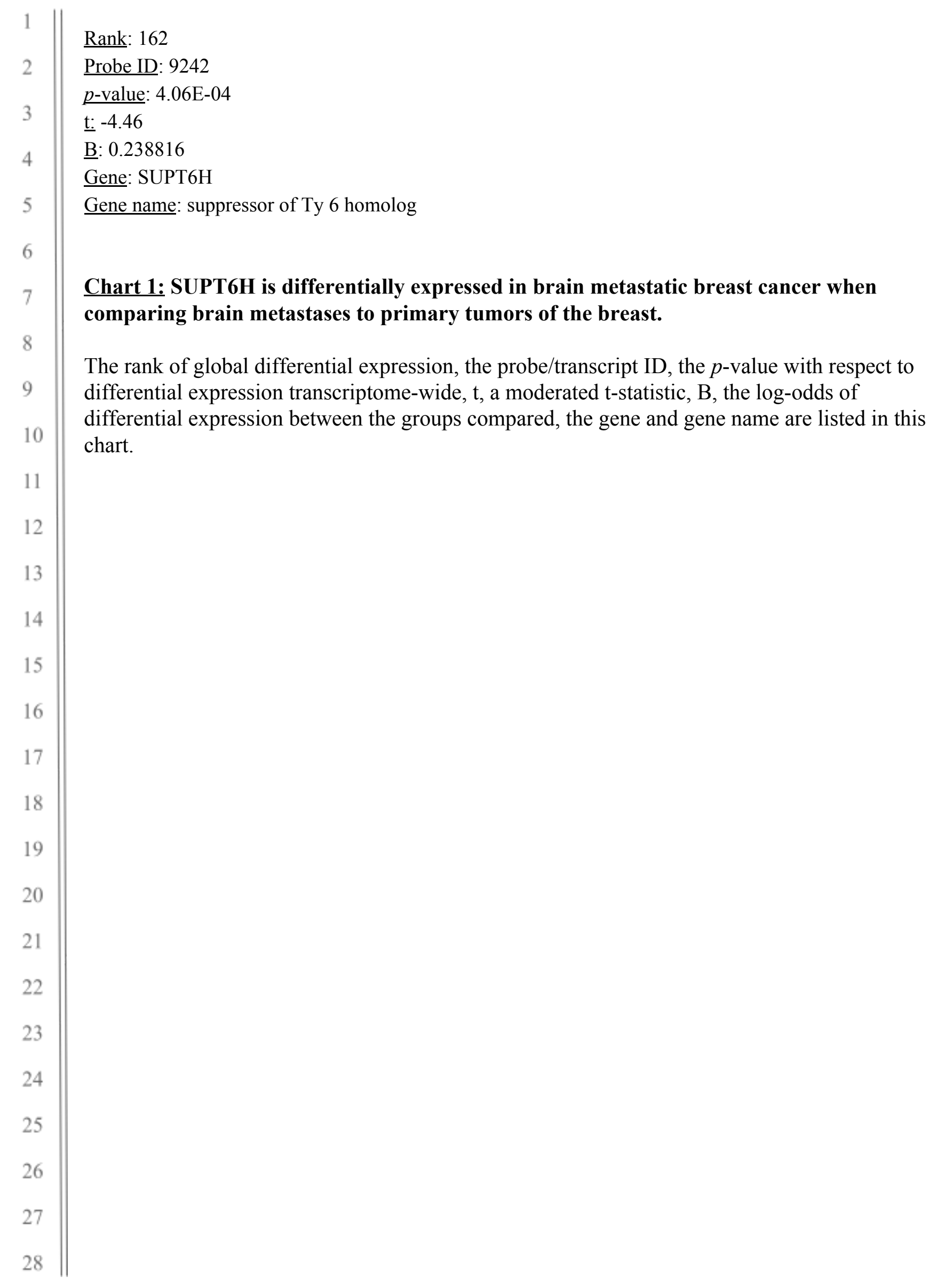




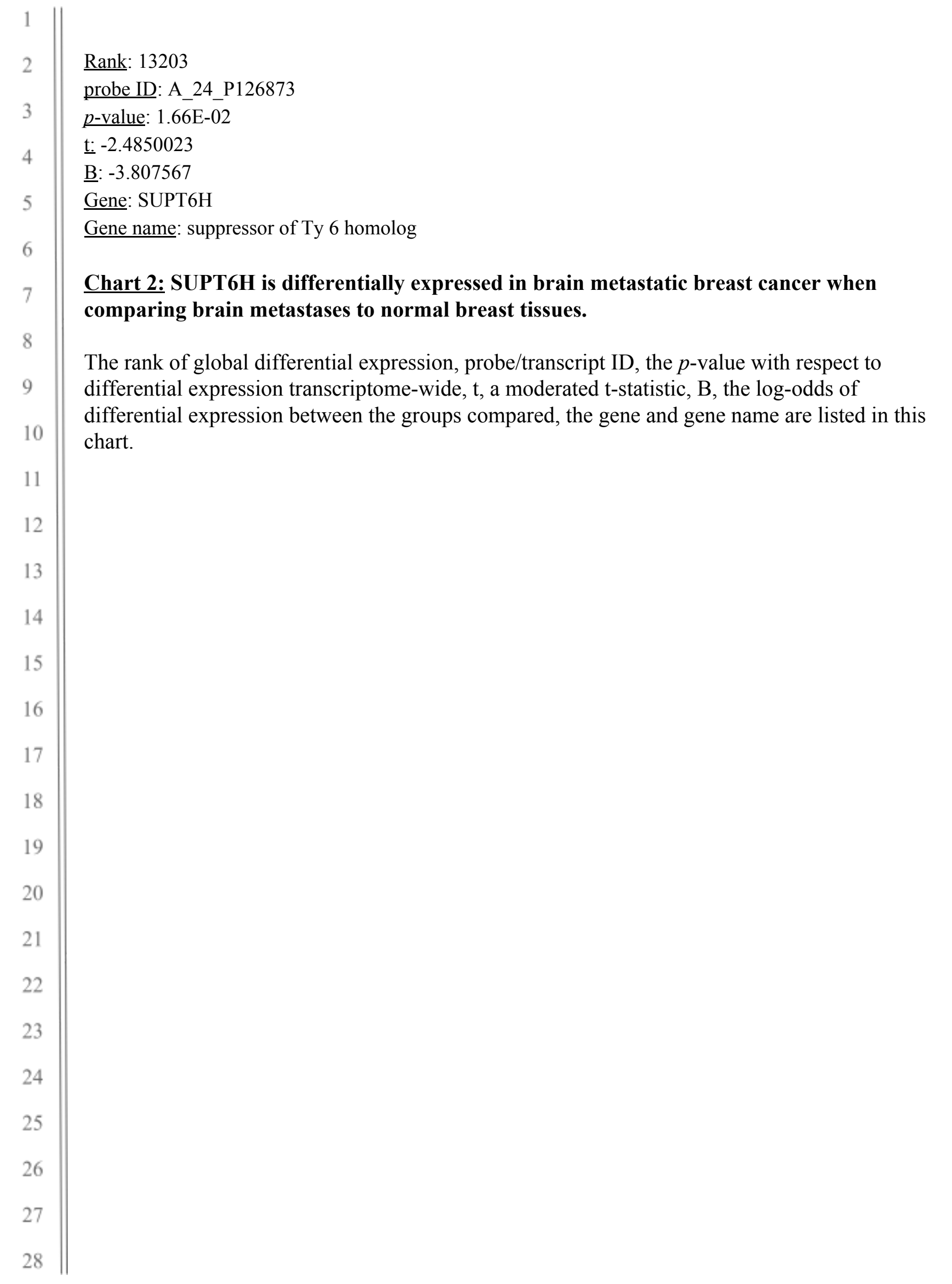




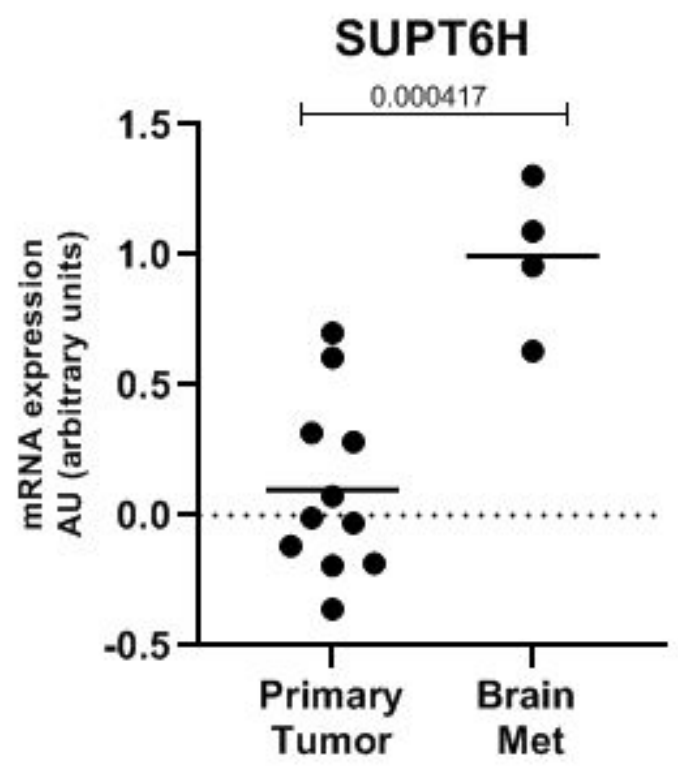

Figure 1: SUPT6H is expressed at significantly higher levels in the brain metastases of patients with metastatic breast cancer when compared to primary tumors of the breast.

The mRNA expression level of SUPT6H in primary tumors of the breast (left) and in brain metastases of women with metastatic breast cancer (right) is graphically depicted; the result of a statistical test evaluating significance of difference in SUPT6H expression between primary tumors of the breast and brain metastases, a $p$-value, is $p=0.000417$. 


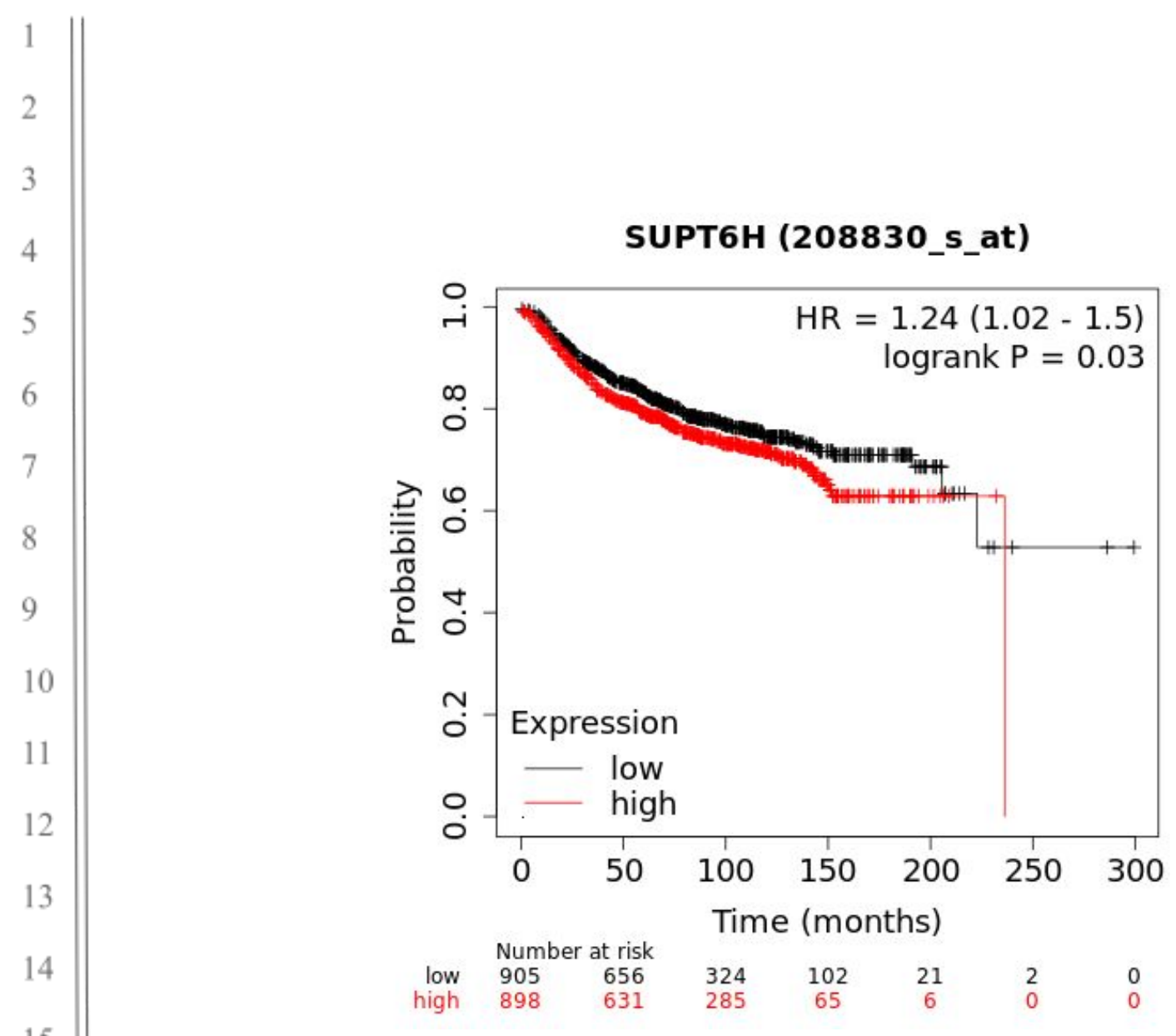

Figure 2: Significant correlation between SUPT6H primary tumor expression and distant metastasis-free survival in patients with breast cancer.

Depicted in this Kaplan-Meier plot is the probability of distant metastasis-free survival for $n=1803$ total patients stratified into two groups, based on low or high expression of SUPT6H in patient primary tumors. The log rank $p$-value denoting statistical significance of difference in distant metastasis-free survival when comparing the two groups, as well as hazard ratio for this comparison is listed above. Listed below is the number of patients at risk (number of patients alive) per interval, after stratification based on SUPT6H expression; in the first interval, number at risk is number of patients alive; in each subsequent interval, number at risk is the number at risk less those who have expired or are censored. 


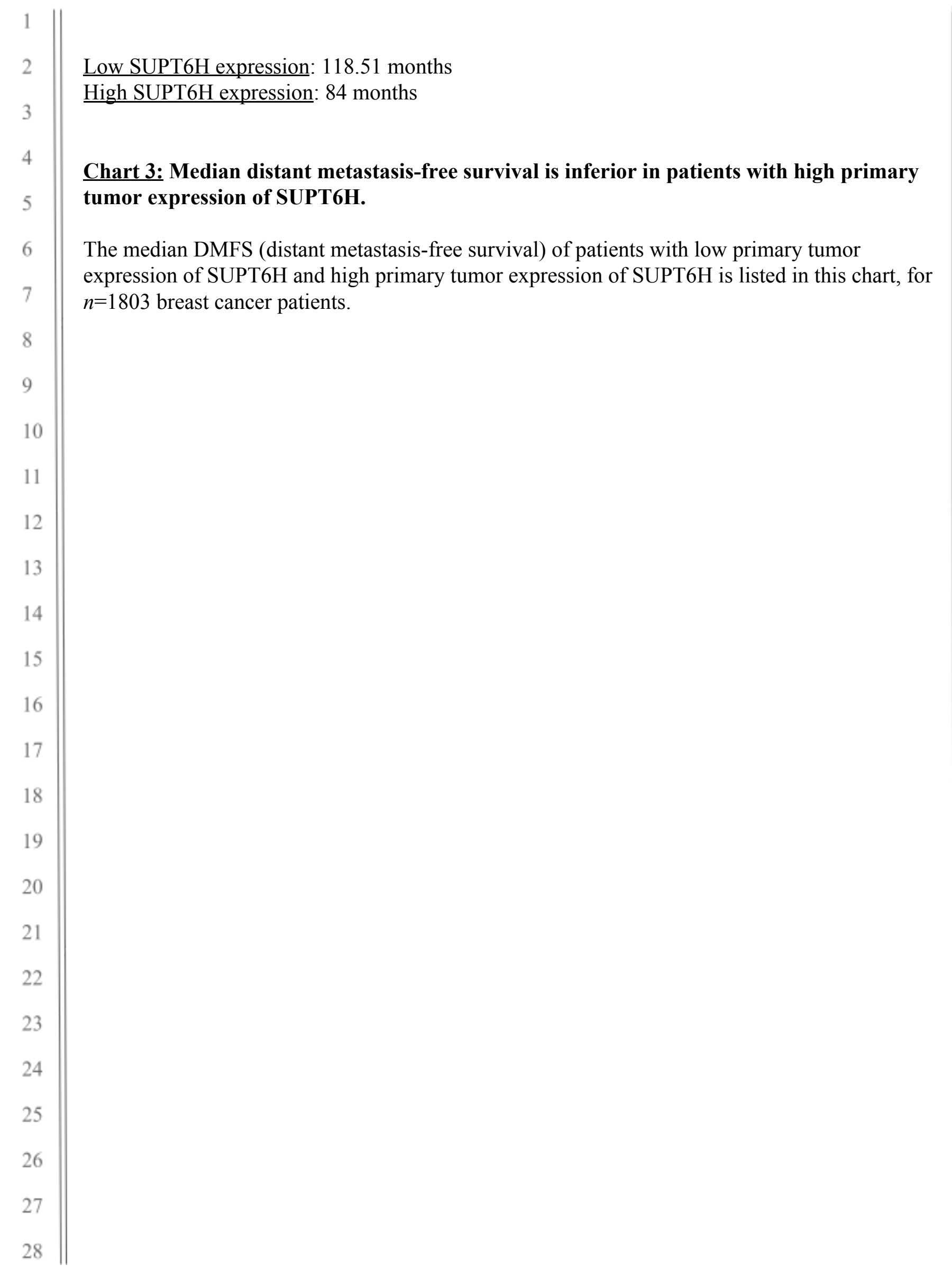

